\title{
Activity Recognition for Emergency Care using RFID
}

\author{
Siddika Parlak \\ Rutgers University \\ New Brunswick, NJ 08854 \\ parlak@rci.rutgers.edu
}

\author{
Ivan Marsic \\ Rutgers University \\ New Brunswick, NJ 08854 \\ marsic@ece.rutgers.edu
}

\author{
Randall S. Burd \\ Children's National Medical Center \\ Washington, DC 20010 \\ rburd@childrensnational.org
}

\begin{abstract}
We present a system that recognizes human activities during trauma resuscitation, the fast-paced and team-based initial management of injured patients in the emergency department. Most objects used in trauma resuscitation are uniquely associated with tasks. To detect object use, we employed passive radio frequency identification (RFID) for their size and cost advantages. We designed the system setup to ensure the effectiveness of passive tags in such a complex setting, which includes various objects and significant human motion. Through our studies conducted at a Level 1 trauma center, we learned that objects used in trauma resuscitation need to be tagged differently because of their size, shape, and material composition. Based on this insight, we classified the medical items into groups based on usage and other characteristics. Objects in different groups are tagged differently and their data is processed differently. We applied machine-learning algorithms to identify object-state changes and process the RFID data using algorithms specific to object groups. Our results show that RFID has significant potential for automatic detection of object usage in complex and fast-paced settings.
\end{abstract}

\section{Categories and Subject Descriptors}

H.4 [Information Systems Applications]: Miscellaneous; J.3 [Life and Medical Sciences]: Health

\section{General Terms}

Design, Experimentation.

\section{Keywords}

RFID, activity recognition, object sensing, trauma resuscitation.

\section{INTRODUCTION}

Trauma is an important cause of disability and mortality in children and young adults. When patients are severely injured, the initial care in the emergency department, termed "trauma resuscitation" is guided by a defined sequence of evaluation and treatment steps to ensure timely and efficient care. Deviations from this protocol can lead to potentially adverse outcomes. Although some errors have no direct impact on patient, others

Permission to make digital or hard copies of all or part of this work for personal or classroom use is granted without fee provided that copies are not made or distributed for profit or commercial advantage and that copies bear this notice and the full citation on the first page. To copy otherwise, to republish, to post on servers or to redistribute to lists, requires prior specific permission and/or a fee.

BODYNETS 2011, November 07-08, Beijing, People's Republic of China Copyright (c) 2012 ICST 978-1-936968-29-9

DOI 10.4108/icst.bodynets.2011.247213 might directly contribute to poor outcome, or even death [5].

In this work, we propose a system for automatic recognition of the activities performed during trauma resuscitation. Our system consists of a hardware setup (RFID tags and readers) for sensing the environment and algorithms for processing the sensor readings. When recognizing activities, our focus is on the key tasks that should be included in all resuscitations and that could lead to adverse outcomes if omitted or improperly performed. Our system can be used for real-time capturing and displaying of situational information, providing decision support to trauma errors, and for documenting the process.

The tasks performed during resuscitation are complex activities, comprising many simple body movements (walking, raising the arm, hand manipulation), and interactions with medical tools and the patient. Although simple activities can be recognized by sensing the human motion by computer vision or on-body sensors, complex activities require other cues such as spoken words, location, or used tools. Given that most medical tasks are uniquely associated with the used tools, knowing the set of tools in use is crucial for activity recognition. The trauma resuscitation context further limits the possible number of activities. For example, although the handling of a blood pressure (BP) cuff may imply blood pressure measurement, it may also imply restocking or inventorying medical equipment. In the context of resuscitation, the use of a BP cuff for blood pressure measurement is the only relevant and other tasks are unlikely to happen. Assuming that the used objects are correctly identified, most of the trauma tasks can be inferred using existing methods [12][13]. This paper focuses on the key challenge of object-based activity inference for trauma resuscitation: the accurate detection of the used objects.

\subsection{Challenges of Object-Use Detection in a Trauma Setting}

Accurate automatic identification of the used objects is difficult in the trauma setting compared to other domains, such as activities of daily living (ADL). The key domain-specific challenges are:

Teamwork: Tasks are often performed collaboratively and in parallel, involving multiple objects. Hence, teamwork introduces potential ambiguities in object-use detection. For example, occlusions create problems for vision algorithms, and radio interference of multiple sensors and other objects, including people, are challenge to wireless item-based sensors.

Environmental Dynamics: Team members are in motion and large objects, such as the X-ray machine, are moved during resuscitation, thus frequently changing the environment properties relating to the sensed radio signal or visual appearance. 
Lack of Tolerance for Distractions: In stressful settings sensors must be minimally obtrusive. People may avoid bulky sensors or forget performing an action if sensing entails active participation.

Task Uncertainty: During a trauma event, patient history is usually unavailable. Patient management relies on emerging rather than existing information.

Object Characteristics: Trauma tools have several characteristics that must be considered:

- Orientation: Most of the objects do not remain in a fixed orientation, representing a challenge for vision-based algorithms and passive sensors.

- Packaging: Many medical items are sterilized or produced in wrappings. The removal of packaging does not necessarily signal item usage. Also, after the packaging is removed, any sensors attached to it cannot be used to track the object itself.

- Amount: Some tools in the trauma bay occur in large numbers, such as syringes, endotracheal (ET) tubes and IV catheters. An inexpensive and efficient solution is needed to monitor these kinds of items.

- Disposability: Because of sterilization and packaging, many items are not reusable. Attached sensors must be inexpensive enough to be disposable.

- Material: Medical items are composed of various materials such as plastic (e.g., chest tube, ET tube, face mask), rubber (e.g. tourniquet, parts of the BP cuff), metal (e.g., laryngoscope) and even liquids (saline fluid, antiseptic solutions). The sensor technology must be able to handle various background materials.

- Trays/kits: Items that are used together to perform a task, such as chest tube insertion or Foley catheter insertion, are grouped in a tray or kit. It might be feasible to attach sensors only to these trays and kits.

\subsection{Related Work}

To date, object-based activity recognition research has mostly focused on recognizing daily activities performed in a relaxed environment that tolerates variations in sensor size, on-body sensors and near-field technologies [7][12][13]. When recognizing medical activities, such as hospital workers' activities [4][14] and phases of an operation [2][9], object information was often collected manually [4][14] or using near-field RFID technology with wearable readers [2]. Passive RFID tags have been scanned at a designated RFID reader to identify the administered medications and to detect medically significant events, such as administration of anesthesia [1]. Similarly, carts equipped with RFID readers have been used at the point-of-care for detecting administered medications [8].

In contrast to these studies, our system must be able to collect the object data automatically without human effort. The system must also allow for long-term operation in actual trauma bay without disrupting trauma team activities. To achieve this goal and meet the challenges listed earlier, we used passive RFID technology, which is battery-less (which reduces the tag cost and size) minimally intrusive, suitable for irregular object shapes and, unlike computer vision, preserves privacy. Passive RFID tags, however, are sensitive to orientation and the background material: very poor reception is observed when the tag is perpendicular to the antenna, or attached to metallic items or fluid containers. Special tags for metals and liquids are now commercially available. Also, by tagging trays or kits, it may not be necessary to tag a metallic item inside (e.g., scalpel in a chest-tube tray). Because the received RFID data are noisy, we used machinelearning methods and the observed characteristics of trauma teamwork to detect the interactions with the tagged medical tools.

The remainder of this paper is organized as follows. Section 2 describes the RFID equipment setup and our methodology for processing the RFID data. Experimental setup and results are presented in Section 3, along with the findings and discussion of the results. Our conclusions are presented in Section 4.

\section{ACTIVITY RECOGNITION USING PASSIVE RFID TAGS}

Our system consists of three layers concerned with increasing level of abstraction for activity recognition (Figure 1). At the lowest layer (sensing), RFID readers periodically interrogate the tags and pass the raw data to a central computer. Next, a sequence of used objects is inferred from the readouts from all tags. Object usage is decided by detecting changes in the RFID signal state, through mobility detection and localization. The false alarms are removed based on the knowledge of interaction characteristics to obtain actual usage intervals (object detection). At the highest layer, the sequence of used objects is processed to infer performed tasks (activity recognition).

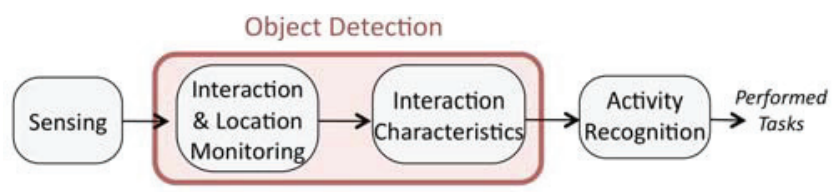

Figure 1: Activity recognition system architecture.

\subsection{RFID-based Sensing}

\subsubsection{RFID Tag Placement}

We decided the tag type for each object to be tracked based on its material and shape, and the type of user interaction. To optimally place the tags on objects, we classified the medical equipment based on interaction characteristics [11]:

- Long-interaction items: When in-use, these items are interacted with for a long time by either the patient or a team member (e.g., cervical collar, thermometer). We attach two tags to such items: one at the point of contact with human body (to detect interaction) and one where it is likely to remain exposed to RF signal, to localize it and detect motion.

- Short-interaction items: These items are interacted with only briefly; therefore, it may not be possible to detect the exact instant of contact. We use a single tag per item to determine its location and mobility. Kits and trays also belong to this class, in which case multiple items are associated with a single tag.

\subsubsection{RFID Antenna Positioning}

We positioned the RFID antennas in the trauma bay by considering the location of large objects and the distribution of medical tools. In a typical trauma bay, a patient bed is at the center; cabinets, benches, drawers and small tables are next to the left, right and head walls (Figure 2). The foot of the bed is usually kept empty for easy accessibility. Medical tools are stored on or in these furniture items or, when in-use, close to the patient bed. It is possible to define four primary zones where the objects lie: central, right, left, and head zones (Figure 2) [11]. 


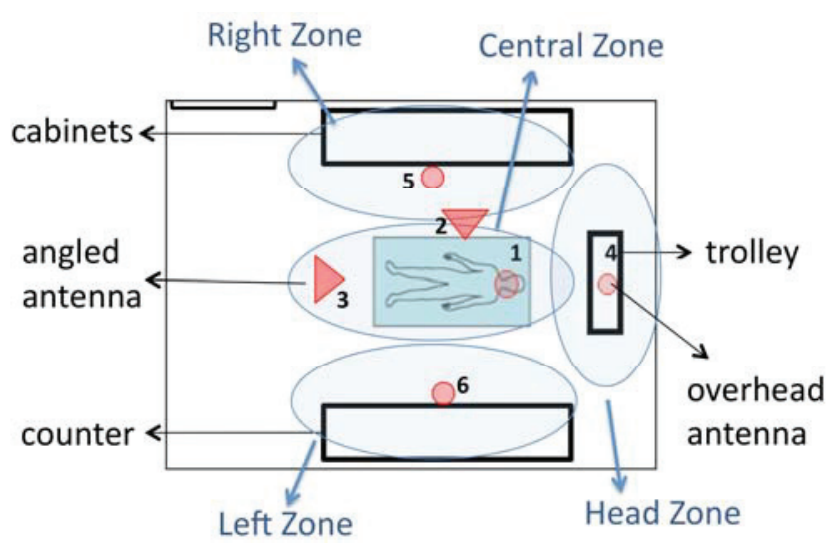

Figure 2: The experimental floor plan.

To reduce the effects of human presence and movement on the system, we placed the antennas above the left, right, head, and central zones, mounted at the ceiling and facing the floor $(2.7 \mathrm{~m}$ above the floor, shown as circles in Figure 2). Two additional antennas were placed for scanning the central zone to improve readout rates, as well as localization and mobility detection accuracy (shown as triangles in Figure 2). These two antennas were positioned facing the central area, slanted at a 45 ? angle and $2 \mathrm{~m}$ above the floor to avoid obstruction by human motion.

The zone definitions and the zone-based positioning of the antennas are not specific to our setting and can be practiced in other trauma bays and surgery rooms with small adjustments. For example, in a smaller trauma bay, the overhead antennas can be attenuated to minimize the overlap between the antennas' scanning regions. Our findings are, therefore, generalizable once the zones are identified and each covered by at least one antenna.

\subsubsection{RFID Reader Coordination}

We used two readers and six antennas from Alien Technology (Morgan Hill, CA) to build our system. The software running on the readers is the vendor's SDK, modified to scan the environment continuously at given intervals. An application on a host computer listened for notifications from each reader, carrying any tag data that it has read. The RFID readers operate in parallel, but each reader needs to cycle through its active antenna ports in a round-robin fashion. In our experiments, each reader visited its three ports in sequence and transmitted for one second through each port. The reader-antenna connections were arranged to minimize the mutual interference: antennas \#1, \#2 and \#3 were connected to $1^{\text {st }}, 2^{\text {nd }}$ and $3^{\text {rd }}$ ports of reader- 1 and antennas \#4, \#5 and \#6 were connected to $1^{\text {st }}, 2^{\text {nd }}$ and $3^{\text {rd }}$ ports of reader-2 (Figure $2)$. Antennas were therefore active sequentially in pairs $(1,4)$, $(2,5)$, and $(3,6)$. This schedule ensured that the three antennas scanning the central zone were never active at the same time.

\subsection{Object Detection}

Mobility and location information are two important cues that indicate whether a medical item is in use. If an item is near or on the patient bed and moving, the item is likely being used for patient care. Although one or both of the location and mobility cues are usually sufficient, there are exceptions. For example, a stethoscope can be considered as a personal item that is carried by individual members of the trauma team. During the resuscitation, the stethoscope is usually carried around the neck and is moving as the person moves. Given that the team members are usually gathered around the patient bed, stethoscopes are usually located close to the patient bed, even if they are not being used for auscultation. Location and mobility information are therefore not sufficient to detect the usage of a stethoscope.

In addition, an interacted item is not necessarily in-use. For example, when a nurse fetches a thermometer, this action is not always immediately followed by measuring the patient's temperature. If the patient has a face mask for oxygen administration, the nurse leaves the thermometer on the patient bed for later use. At an appropriate moment, the nurse uses the thermometer to measure the temperature. In this case, only the second interaction represents actual usage.

\subsubsection{Mobility Detection}

Here, we first describe mobility detection and localization algorithms. Then we explain how we handle exceptions by exploiting the domain knowledge about the interaction characteristics for different items. Our mobility detection algorithm is based on comparing descriptive statistics of the RSSI values in consecutive sliding windows. The statistics obtained from two consecutive windows are expected to differ significantly when the object is mobile. A simple thresholding strategy is not adequate because the signal strength varies depending on factors such as object- and tag type. For these reasons, we trained a Bayesian classifier for detecting mobility events.

We use the following descriptive statistics:

- Difference of the average RSSI values between two consecutive windows: This number represents how much the signal strengths differ across consecutive windows. Smaller differences indicate less movement. This statistic is calculated separately for each of the six antennas.

- Spearman rank correlation coefficient between two consecutive windows: This number represents how closely ranked the RSSI values are from different antennas in consecutive windows. Higher similarity in the ranking indicates less movement.

- Number of antennas that are common for two consecutive windows: This number represents the similarity of two consecutive windows in terms of the number of antennas that successfully receive RSSI signals in both windows.

These statistics are joined into an eight-dimensional feature vector. This feature vector is input to the Bayesian classifier to determine if the tag is moving. The classifier output is smoothed by a Gaussian filter to remove spurious fluctuations. Lastly, a binary decision (moving or not) is made for each point in time.

\subsubsection{Localization}

In the localization step, our aim is to identify the zone (Figure 2) where the object lies. If the object is on or near the patient bed (central zone), it is highly likely to be in use. The RSSI signal received from a tag reveals its proximity to a reader antenna. Because each zone is covered with a mutually exclusive set of antennas, the zone scanned by the antenna with the maximum RSSI is taken to be the tag's location. Although it is possible to use more complex approaches, as well as finer-grained localization algorithms [11], this zone-based localization is adequate for our envisioned applications in the trauma resuscitation domain (mentioned in Section 1).

\subsubsection{Filtering Based on Interaction Characteristics}

Item interaction and motion do not necessarily imply its usage for trauma resuscitation purposes. In addition to accidental and spurious interactions, personal tools, such as stethoscope, may cause high rate of false alarms. To reduce false alarms when 


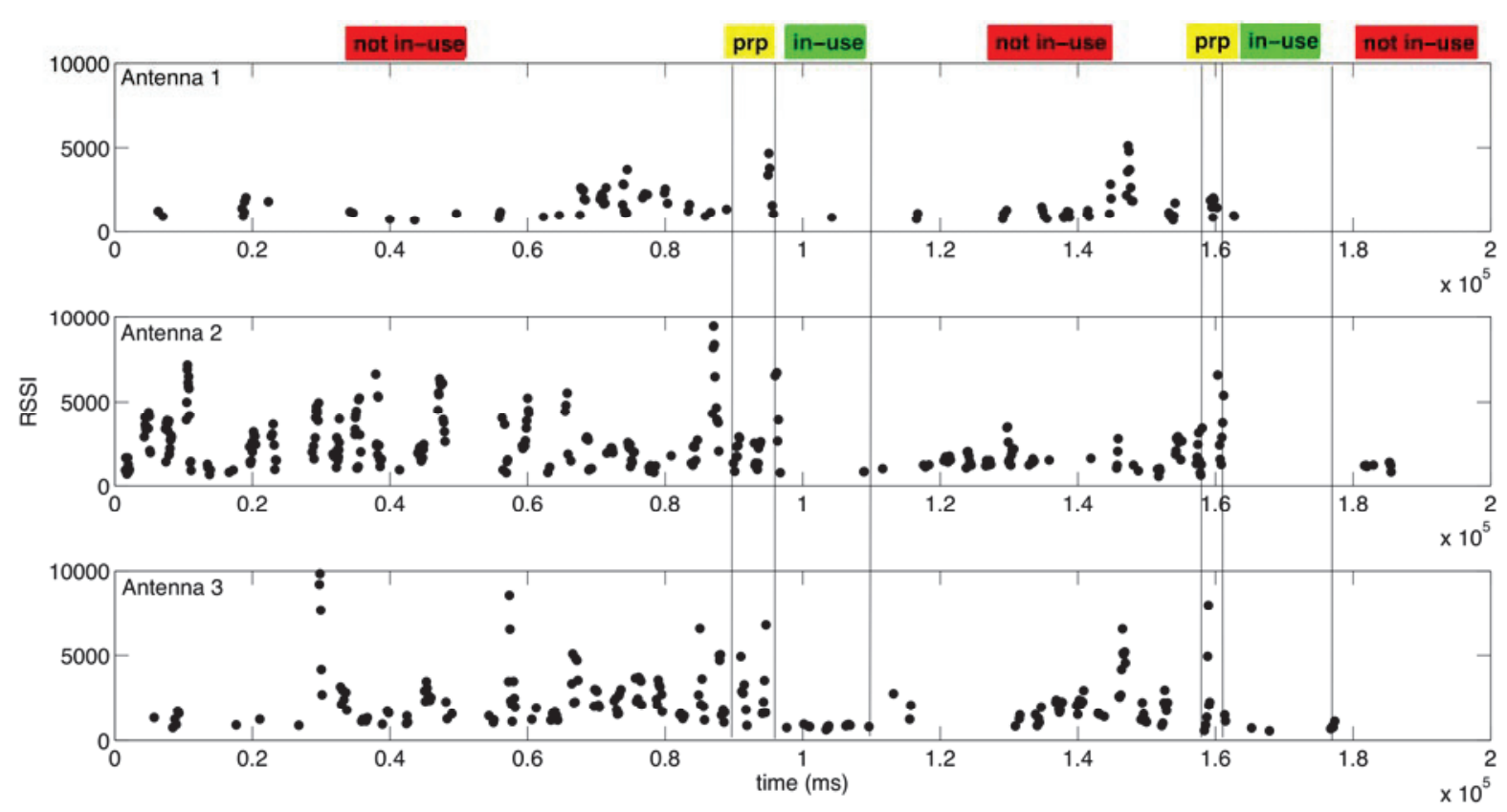

Figure 3: RSSI values for the tag attached to the stem of the stethoscope, captured by three antennas during a simulated resuscitation. Vertical lines indicate transitions between states of the stethoscope. ("prp" = preparation).

detecting the long-interacted items, we apply a filtering approach. As mentioned, long-interacted items were tagged with two tags: one at the point of contact with human body (to detect interaction) and one at a location where it is most exposed to radio signal (to localize it and detect motion). Our filtering algorithm uses the RSSI signal from both tags to decide whether a change in object state signals an actual use. If the RSSI from the first (interactiondetection) tag is low or none, and the RSSI from the second (localization+motion-detection) tag is high, this instance of state change is retained. Otherwise, the change is discarded. Figure 3 shows RSSI values received from an RFID tag attached to the stethoscope stem. Note that when the stethoscope is in-use, either no readings or very weak RSSI values are observed from this tag.

We also applied thresholding on the posterior probabilities of the classifier output. In addition, detected fluctuations that are shorter than the expected duration of interaction are filtered out.

\subsection{Activity Recognition}

When the activity recognition is limited to trauma resuscitation tasks, many tasks can be inferred unambiguously from the used objects. For instance, if the BP cuff is being used during an event, we can infer the current activity as manual BP measurement. Likewise, a thermometer use implies temperature measurement, a chest-tube-tray use implies chest-tube insertion and a laryngoscope use implies endotracheal intubation. In some cases, an object use does not uniquely identify a task. For instance, a stethoscope can be used both for listening breath sounds and assessing circulatory dynamics, while an otoscope can be used to assess pupils as well as assessing the external auditory canal.

Most of medical equipment is uniquely associated with tasks. However, multiple tools may be needed to perform a single task. When several tools are used in the same task, it suffices to detect the use of at least one of these tools with high confidence. For example, the use of either $\mathrm{CO}_{2}$ indicator, ET tube, or laryngoscope, signifies the task of intubation. However, these tools are used at different times during intubation, so the intubation interval will not be correctly determined if the use of all three tools is not correctly detected. We would know that an intubation task was performed, but we would not know when exactly it started or finished. We could detect the task extent by fusing use-detection of all tools associated with a task.

Activity recognition requires the knowledge of whether a tool is being used and when. Both of these are derived based on detecting state changes for tools. When a tool is relocated from a storage place to the patient bed, in most cases it will be used in the resuscitation. The greatest gain in determining whether a tool will be used is therefore obtained from detecting the relocation of the tool. If the tool is wrapped in packaging, the removal of the packaging reinforces the likelihood that the tool will be used. This gain is relatively small and it is very difficult to detect the action of packaging removal. As seen in Figure 4, the change in RSSI signals is much greater for item relocation than for unwrapping. The key problem is that there will be many spurious interactions. For example, after the object is relocated to the bed, but before it is unwrapped, it may be accidentally disturbed. After the wrapping is removed, it is usually left on the bed and it too can be accidentally disturbed. These disturbances are difficult to distinguish from wrapping removal using only RSSI signals. In the timeline in Figure 5, the last three interactions with the ETtube tag at 8th and 9th minutes represent handling the empty wrapping (because the wrapping was removed during 4th minute).

For detecting when a tool is in-use, we observed that in actual resuscitations most of the time the tool will be used soon after relocation. The key gain is from detecting relocation, and detecting the moment of unwrapping is not very beneficial considering the difficulty of its detection. Therefore, in this paper, we focus on detecting the relocation event and, based on it, decide whether and when the tool is in use.

An additional difficulty with detecting object usage arises because some objects are returned back to the storage place after usage, while other objects remain on the patient bed. For example laryngoscope, thermometer and otoscope are returned back to the storage after use, but $\mathrm{CO}_{2}$ indicator, IV kit and ET tube are usually left on the bed along with their wrapping (which contains the tag). Because the latter objects are left in the working area, 


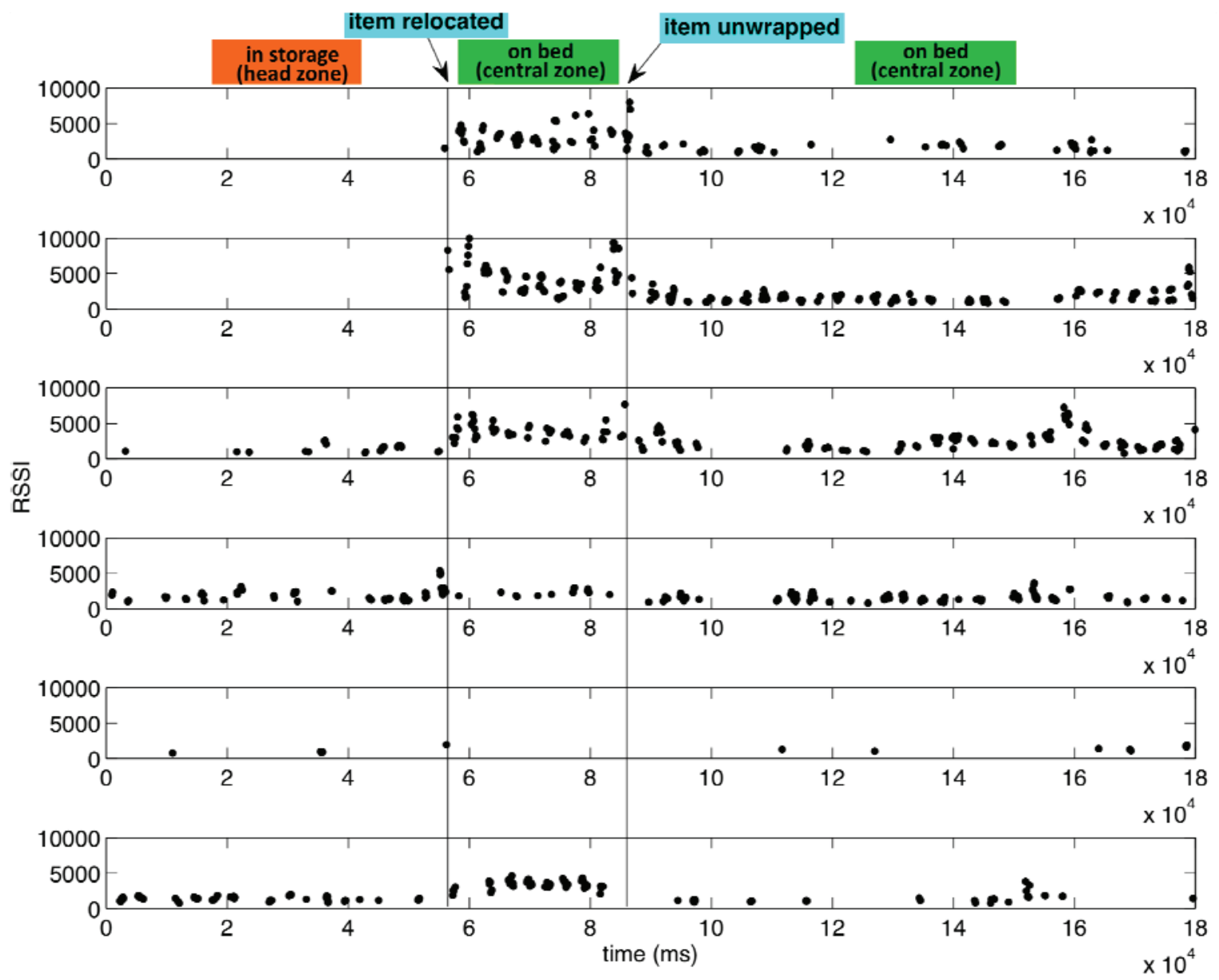

Figure 4: RSSI values from the tag attached to the $\mathrm{CO}_{2}$ indicator, captured by six antennas during a simulated resuscitation. Vertical lines indicate transitions between states of the $\mathrm{CO}_{2}$ indicator.

there will be accidental interactions or occlusions that produce false alarms about their state change and, in turn, their usage.

\section{EXPERIMENTS}

\subsection{Experimental Setup}

\subsubsection{Activity List for Recognition}

We began our analysis with a list of tasks that are important for the performance of trauma resuscitation. This list was prepared by a group of six physicians (emergency medicine, anesthesiology, and trauma surgery), two nurses, and two respiratory therapists at Children's National Medical Center. These tasks were judged as key steps that should be included in all resuscitations, for their omission or incorrect ordering could lead to potentially adverse outcomes. We focused on the primary survey and selected 8 tasks for detection. Activities for the setup of vital-sign instruments are not included, because they can be detected when the physiological signals become available. The tasks and the related tools are listed in Table 1. Tagged items are shown in Figure 6.

\subsubsection{Data}

The dataset was recorded in 15 simulated resuscitations performed in our laboratory. In each session, one experimenter performed the activities in Table 1 by interacting with the associated medical tool, one experimenter played the role of a patient, and a third experimenter recorded the video. The experimenter performed the activities sequentially, one immediately after another. Using this approach, a continuous set of activities is obtained, rather than an isolated set. The total duration of a session varied, with an average of 3.5 minutes. The experiment was performed with five different participants, each repeating the experiment for three times. The participants were not trained in using the tools before the experiment. They were not aware that they should cover one of the tags during usage, in cases where we used two tags on a tool.

We designed the experimental scenario and the environmental setting by reviewing real and simulated resuscitation videos recorded at our collaborating hospital. The experimental scenario includes the activities listed in Table 1, as well as spurious interactions to create false alarms. The approximate duration of

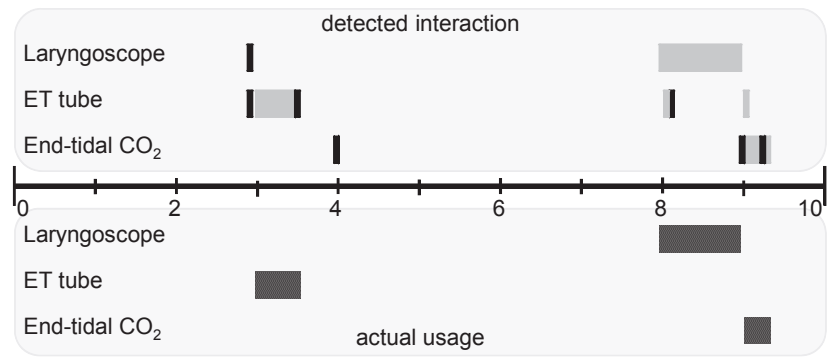

Figure 5: Timeline of an intubation event. The top half of the chart represents interactions detected by RFID and the bottom half represents the actual usage observed in a recorded video.

Black bars represent linear movement, gray bars represent random movement, and dotted bars represents actual usage. 


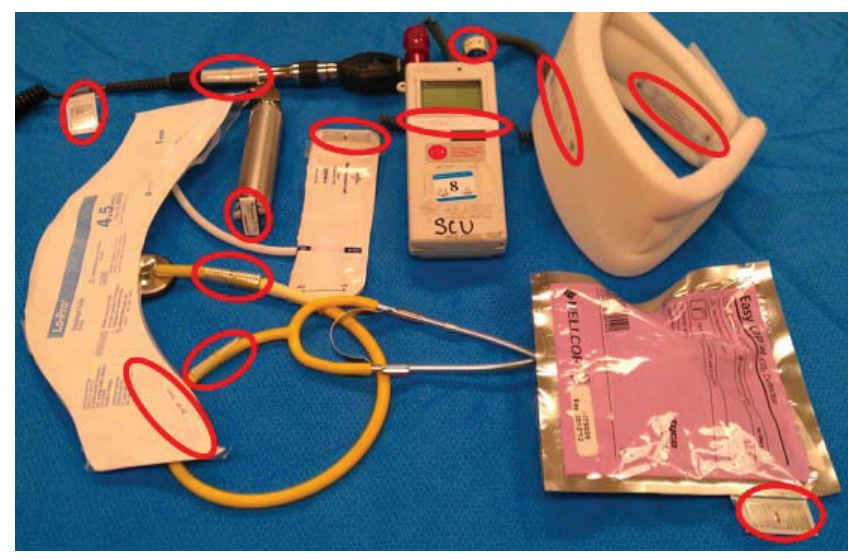

Figure 6: RFID-tagged medical items for the experimental evaluation. The tags are circled.

activities was extracted from these videos and experimenters were asked to perform each activity in the specified time interval. The environmental setting was designed to replicate a real trauma bay (Figure 2), and the medical objects were located in storage places identified from videos of actual resuscitations. All objects were in the reading zone of at least one RFID reader throughout the session, even when they were not in use. At each time instant, the algorithm processed RSSI readings coming from all objects and determined whether each object is in use.

\subsubsection{Metrics}

Object detection performance was evaluated with these metrics:

$$
\begin{gathered}
\text { Precision }=\frac{\text { true positive }}{\text { true positive }+ \text { false positive }} \\
\text { Recall }=\frac{\text { true positive }}{\text { true positive }+ \text { false negative }}
\end{gathered}
$$

A hypothesized detection event is declared correct if it is in the 10 -second neighborhood of the actual interaction. Multiple detections in the neighborhood of the same interaction are considered as a single detection.

\subsection{Experimental Results}

Our experimental results (Table 2) show that most of the tool interaction events were located correctly in time. We also checked the metrics for individual participants and found that they are approximately same. An average precision rate of $63.8 \%$ in Table 2 indicates that one third of detection events are false alarms. As discussed in Section 2.3, for tasks that require the use of several tools, these false alarms can be eliminated if the use of some tools can be detected with high confidence.

We next analyzed how detection performance varies depending on each medical tool. Table 2 shows that the recall rate is around $90 \%$ for most tools. The low precision rates of the $\mathrm{CO}_{2}$ indicator and the IV kit are due to their positions after usage. As discussed in Section 2.3, item packaging for these objects is not thrown away immediately after removal (and, presumably, the item is used). Because the team members are busy with patient care, packaging is often left on the patient bed for later disposal. As they continue with the resuscitation, team members will occlude or accidentally interact with objects or packaging. False alarm rates are therefore high for these types of objects.

In case of the thermometer, the probe surface is relatively small compared to other tagged surfaces. To tackle this drawback, we folded the RFID tag around the probe, which impaired the radio signal reflected from the tag. Although the thermometer is interacted with for a long time, the exact instant of interaction may be missed. High false-alarm rate for the otoscope is due to its storage position, which is in between central, right, and head zones. Because this area is not adequately covered by any of the antennas, the signal strength values are low and the ranking of antennas (by the received signal strength) may quickly change. As a result, these fluctuations are mistakenly detected as interactions.

\subsection{Discussion}

In our experiments, we used several types of UHF passive RFID tags from different vendors, including an on-metal tag. We did not observe a significant effect of the tag type on object detection performance. Success of object detection mostly depended on the duration of interaction, shape/size of the tool, and tool handling habits of clinicians (e.g., leaving packaging on the bed after unwrapping a tool). While our approach of attaching two tags to the long-interacted items was generally helpful, it had limitations when the contact surface was small (e.g., for the thermometer).

A limitation of object-based methods is the inability to recognize activities involving a few or no objects. In resuscitation, manual palpations and spoken assessments are common non-instrumental tasks. A potential solution is to use vision or motion detectors.

When the same item is used to perform consecutive tasks without a break, an object-based recognizer is unable to distinguish these

Table 1: Set of tasks for activity recognition, generated based on the essential activities during the primary survey in trauma resuscitation. Useful information for object detection is represented with $M$ (motion), L (location), and I (interaction).

\begin{tabular}{|l|l|l|l|l|l|}
\hline Activity & Associated tool & Taggable part & Tag type & Interaction & Useful info \\
\hline \hline Immobilize neck & Cervical collar & itself & regular & long & $\mathrm{M}, \mathrm{L}, \mathrm{I}$ \\
\hline \multirow{3}{*}{ Establish airway } & Laryngoscope & itself & packaging & on-metal & long \\
& regular \\
short & $\mathrm{CO}_{2}$ indicator & packaging & short & $\begin{array}{l}\mathrm{M}, \mathrm{L} \\
\mathrm{M}, \mathrm{L} \\
\mathrm{M}, \mathrm{L}\end{array}$ \\
\hline Listen to breath sounds & Stethoscope & itself & regular & long & $\mathrm{I}$ \\
\hline Measure blood pressure & BP cuff & itself & regular & long & $\mathrm{M}, \mathrm{L}, \mathrm{I}$ \\
\hline Measure temperature & Thermometer & itself & regular & long & $\mathrm{M}, \mathrm{L}, \mathrm{I}$ \\
\hline Establish IV access & IV kit & packaging & regular & short & $\mathrm{M}, \mathrm{L}$ \\
\hline Check pupils and ears & Otoscope & itself & regular & long & $\mathrm{M}, \mathrm{L}, \mathrm{I}$ \\
\hline Roll patient & Patient bed & itself & regular & long & $\mathrm{I}$ \\
\hline
\end{tabular}


Table 2: Motion-detection performance for different medical tools

\begin{tabular}{|l|c|c|}
\hline Object & Precision (\%) & Recall (\%) \\
\hline \hline Cervical collar & 100 & 90 \\
\hline Laryngoscope & 69.2 & 90 \\
\hline Endotracheal tube & 66.6 & 100 \\
\hline $\mathrm{CO}_{2}$ indicator & 42.9 & 90 \\
\hline Stethoscope & 77.3 & 89.5 \\
\hline BP cuff & 90.9 & 100 \\
\hline Thermometer & 40.9 & 100 \\
\hline IV kit & 54.6 & 60 \\
\hline Otoscope & 58.8 & 100 \\
\hline Patient bed & 72.7 & 88.9 \\
\hline Average & $\mathbf{6 3 . 8}$ & $\mathbf{9 0 . 6}$ \\
\hline
\end{tabular}

tasks. We observed this behavior in recorded videos of trauma resuscitations. For example, an otoscope is used to assess ears and an ophthalmoscope is used to assess pupils. However, we observed that a team member usually evaluates both pupils and ear once the otoscope is grasped, and avoids spending time to grab the other device. A potential solution would be to complement RFID sensing with other sensory modalities.

Electromagnetic interference between the UHF RFID system and other devices, which may cause their malfunctioning, is a critical issue in patient care. One study with 246 tests found 68 cases of interference [15], ranging from minor effects (e.g., unexpected noise on the computer monitors) to potentially hazardous failures (e.g., stopped infusion pumps or ventilators), and occurred at distances from $1 \mathrm{~cm}$ to $6 \mathrm{~m}$ away from the interfering device. Another study [3] reported no interference in 1600 tests on five devices at any distances tested $(0.3$ to $1.8 \mathrm{~m})$, claimed to be more likely in practice. In [6], it was deduced that the chance of interference grows with factors ( $i$ ) higher output power of the RFID system; (ii) shorter distance between the RFID reader and the medical device; and, (iii) the presence of a tag on the device.

Our tests performed in the collaborating medical center found no interference with the UHF RFID system on patient monitors used for a patient simulator. During the tests, six antennas were deployed in the room, transmitting sequentially in pairs, with maximum signal strength ( 1 Watt). The closest and farthest antennas were 0.6 and 3.5 meters away, respectively. The system worked for more than an hour without observable interference.

\section{CONCLUSIONS}

We presented an RFID-based system that automatically recognizes high-level human activities during trauma resuscitation. Our system uses object-based activity recognition, exploiting a nearly unambiguous relationship between tasks and medical tools used in those tasks. The key challenge was to identify the used objects in a correct and nonintrusive way. Our results show the feasibility of using RFID technology and machine-learning techniques to achieve this goal.

\section{ACKNOWLEDGMENTS}

We thank to Children's National Medical Center personnel for consenting to participation in experiments, and providing equipment and the activity list.

\section{REFERENCES}

[1] S. Agarwal, A. Joshi, T. Finin, Y. Yesha, and T. Ganous. A pervasive computing system for the operating room of the future. Mobile Net's and Applic's, 12(2-3):215-228, 2007.

[2] J. E. Bardram, A. Doryab, R. M. Jensen, P. M. Lange, K. L. G. Nielsen, and S. T. Petersen. Phase recognition during surgical procedures using embedded and body-worn sensors. Proc. of the IEEE Conf. on Pervasive Computing and Communications (PerCom), page 45-53, Seattle, USA, 2011.

[3] B. Christe, E. Cooney, G. Maggioli, D. Doty, R. Frye, and J. Short. Testing potential interference with RFID usage in the patient care environment. Biomedical Instrumentation \& Technology, 42(6):479-484, November 2008.

[4] J. Favela, M. Tentori, L. Castro, V. Gonzalez, E. Moran, and A. Martinez-Garcia. Activity recognition for context-aware hospital applications: issues and opportunities for the deployment of pervasive networks. Mobile Networks and Applications, 12(2):155-171, 2007.

[5] R. L. Gruen, G. J. Jurkovich, L. K. McIntyre, H. M. Foy, and R. V. Maier. Patterns of errors contributing to trauma mortality: Lessons learned from 2594 deaths. Annals of Surgery, 244(3):371-380, 2006.

[6] B. Houliston, D. Parry, C. S. Webster, and A. F. Merry. Interference with the operation of medical devices resulting from the use of radio frequency identification technology. New Zealand Medical Journal, 122(1297):9-16, June 2009.

[7] B. Logan, J. Healey, M. Philipose, E. Tapia, and S. Intille. A long-term evaluation of sensing modalities for activity recognition. Proc. 9th Int'l Conf. Ubiquitous Computing, pp. 483-500, 2007.

[8] K. Ohashi, S. Ota, L. Ohno-Machado, and H. Tanaka. Smart medical environment at the point of care: Auto-tracking clinical interventions at the bed side using RFID technology. Computers in Biology and Medicine, 40(6):545-554, 2010.

[9] N. Padoy, T. Blum, H. Feussner, M. Berger, and N. Navab. On-line recognition of surgical activity for monitoring in the operating room. Proc. Innov. Applic's of Artific. Intell, 2008.

[10] S. Parlak and I. Marsic. Non-intrusive Localization of Passive RFID Tagged Objects in an Indoor Workplace. IEEE Int'l Conf. RFID-Technologies \& Applic's, Barcelona, 2011.

[11] S. Parlak, A. Sarcevic, I. Marsic, and R. Burd. Introducing RFID technology in dynamic and time-critical medical settings: requirements and challenges. Submitted for review.

[12] D. Patterson, D. Fox, H. Kautz, and M. Philipose. Finegrained activity recognition by aggregating abstract object usage. Proc. 9th IEEE Int'l Symp. Wearable Computers, pp. 44-51, 2005.

[13] M. Philipose, K. Fishkin, M. Perkowitz, D. Patterson, et al. Inferring activities from interactions with objects. IEEE Pervasive Computing, pp. 50-57, 2004.

[14] D. Sánchez, M. Tentori, and J. Favela. Activity recognition for the smart hospital. IEEE Intell. Sys, 23(2):50-57, 2008.

[15] R. van der Togt, E. J. van Lieshout, R. Hensbroek, et al. Electromagnetic interference from radio frequency identification inducing potentially hazardous incidents in critical care medical equipment. Journal of the American Medical Assoc., 299(24):2884-2890, 2008. 